\title{
The Malaria System MicroApp: A New, Mobile Device-Based Tool for Malaria Diagnosis
}

Allisson Dantas Oliveira ${ }^{1,2}$, MSc; Clara Prats ${ }^{3}, \mathrm{PhD}$; Mateu Espasa ${ }^{4}, \mathrm{MD}$; Francesc Zarzuela Serrat ${ }^{4}, \mathrm{MSc}$; Cristina Montañola Sales ${ }^{3}$, PhD; Aroa Silgado ${ }^{4}$, BSc; Daniel Lopez Codina ${ }^{3}$, PhD; Mercia Eliane Arruda ${ }^{5,6}$, PhD; Jordi Gomez i Prat ${ }^{4}, \mathrm{PhD} ;$ Jones Albuquerque ${ }^{1,6}, \mathrm{PhD}$

${ }^{1}$ Federal Rural University of Pernambuco, Department of Statistics and Informatics, Recife, Brazil

${ }^{2}$ Federal University of Rio Grande do Norte, Department of Informatics and Applied Mathematics, Natal, Brazil

${ }^{3}$ Universitat Politècnica de Catalunya, BarcelonaTech, Barcelona, Spain

${ }^{4}$ Vall dHebron University Hospital, Microbiology Department, Barcelona, Spain

${ }^{5}$ Aggeu Magalhães Research Center FIOCRUZ, Recife, Brazil

${ }^{6}$ Keizo Asami Laboratory of Imunopathology, Federal University of Pernambuco, Recife, Brazil

\section{Corresponding Author:}

Allisson Dantas Oliveira, MSc

Federal Rural University of Pernambuco

Department of Statistics and Informatics

Rua Dom Manoel de Medeiros, s/n, Dois Irmãos

Recife, 52171900

Brazil

Phone: 558133206491

Fax: 558133206491

Email: allissondantas@gmail.com

\section{Abstract}

Background: Malaria is a public health problem that affects remote areas worldwide. Climate change has contributed to the problem by allowing for the survival of Anopheles in previously uninhabited areas. As such, several groups have made developing news systems for the automated diagnosis of malaria a priority.

Objective: The objective of this study was to develop a new, automated, mobile device-based diagnostic system for malaria. The system uses Giemsa-stained peripheral blood samples combined with light microscopy to identify the Plasmodium falciparum species in the ring stage of development.

Methods: The system uses image processing and artificial intelligence techniques as well as a known face detection algorithm to identify Plasmodium parasites. The algorithm is based on integral image and haar-like features concepts, and makes use of weak classifiers with adaptive boosting learning. The search scope of the learning algorithm is reduced in the preprocessing step by removing the background around blood cells.

Results: As a proof of concept experiment, the tool was used on 555 malaria-positive and 777 malaria-negative previously-made slides. The accuracy of the system was, on average, $91 \%$, meaning that for every 100 parasite-infected samples, 91 were identified correctly.

Conclusions: Accessibility barriers of low-resource countries can be addressed with low-cost diagnostic tools. Our system, developed for mobile devices (mobile phones and tablets), addresses this by enabling access to health centers in remote communities, and importantly, not depending on extensive malaria expertise or expensive diagnostic detection equipment.

(JMIR Res Protoc 2017;6(4):e70) doi: 10.2196/resprot.6758

\section{KEYWORDS}

artificial intelligence; applied computing; automated diagnosis; malaria; mobile devices 


\section{Introduction}

Malaria is a public health problem worldwide. A total of 214 million cases of malaria occurred globally in 2015 with 438,000 deaths [1]. In 2014, 97 countries reported the continuous transmission of malaria and the World Health Organization (WHO) estimates that around 3.2 billion people are at risk of becoming infected with this disease [1]. In most of the high-burden countries, weak healthcare systems contribute to slower than average declines in malaria incidences and high rates of mortality [1]. Therefore, early and accurate diagnosis of malaria is essential for effective disease management and surveillance. Indeed, misdiagnosis can result in significant morbidity and mortality. In addition, the correct diagnosis of patients with febrile illnesses may help to reduce the emergence and spread of drug resistance by reserving antimalarial treatments for those with a malaria diagnosis.

Malaria is currently diagnosed by microscopy with stained slides (thick blood films or blood smear) or by rapid diagnostic tests (RDTs) [2,3]. The thick blood film method of slide staining is the most common and inexpensive technique to diagnose malaria, whereas, the blood smear method is specifically used to identify the morphology and species of the Plasmodium parasite [4]. Today, it is essential that malaria diagnosis technicians are experienced in identifying species of Plasmodium using these techniques. For this reason, RDT methods are quite effective and widely used in some regions (ie, the Brazilian Amazon). However, RDT methods are expensive and not always effective in identifying samples with mixed species [3,5]. Furthermore, scientists are concerned about parasite resistance to antimalarial medicines and mosquito vector anopheles to insecticides [1]. Thus, a fast, in-place diagnosis system is essential to control malaria.

In recent decades, a number of researchers, including those from computing areas, have sought cost-effective solutions to assist health professionals in the control of epidemics and diseases. For example, Leal Neto et al (2014) developed a real-time diagnostic system for epidemiological events simulations [6]. Medical imaging has also been used successfully in the diagnosis of diseases. Kaewkamnerd and colleagues (2012) developed a 5-phase image analysis system to detect and classify malaria [7]. Techniques, such as hue-saturation-value (HSV) and adaptive threshold, have been used to extract image characteristics and automated systems of image capturing using a motor adapted to a microscope have been proposed [8]. In another study, Anggraini et al (2011) developed an application to successfully separate background of blood cells by solving image segmentation problems [9].
Here, we propose a low-cost, automated diagnostic system for malaria. Digital processing image techniques and a learning process based on artificial intelligence algorithms were combined to develop the system. Prior to app development, training and validation of the classifier were implemented on a personal computer in $\mathrm{C}++$ language with Microsoft Windows 8.1. The minimum requirements for the app were that is used an Android operating system of 4.2 or higher and had a rear camera of at least 5 megapixels (MPs). Therefore, the Galaxy Tab 2 was used for testing. Taking advantage of this computing infrastructure, the system aims to aid public health officials in remote locations by trying to solve pending issues such as accessibility, cost, rapidness, and accuracy in malaria diagnosis.

\section{Methods}

The facial recognition method proposed by Viola and Jones is known as a heuristic method for the robust, fast, and accurate detection of faces in images [10]. Indeed, several studies have demonstrated the application of the technique [11-13]. The Viola and Jones' facial recognition method was used to develop a new method for the representation of images called integral image, which makes use of a simple and efficient classifier using an adaptive boosting learning algorithm [14]. It has also been used in the development of the cascade of classifiers method. This method uses a constant computational cost, which enables its use in real-time applications. The steps of the algorithm will be presented in the following sections.

\section{Haar-Like Features}

Historically, direct pixel manipulation has been a computationally complex problem [15]. As such, Viola and Jones [10] aimed to build a system that could be executed in constant time. They suggested an adaptation of the basic functions of haar described by Papageorgiou and colleagues [16], and began to use haar-like features added to the use of the integral image. Haar-like features are rectangles of white and dark regions. The features value is given by the difference between the sum of the intensities of the pixels of the light region (white) and the sum of pixel intensities in dark region (black) (equation a, Figure 1) where $f(w)$ is a value of feature in the windows $w, \Sigma P_{\text {black }}$ is the sum of pixels in the black region, and $\Sigma P_{\text {white }}$ is the sum of pixels in the white region.

Four basic haar-like features are described to face-detection problems (Figure 2). Each resource-type of haar-like features indicates attendance or absence of features in the image, such as edge detection, texture, and others. The standard detector is used in $24 \times 24$ pixels, generating up to 160,000 rectangle features by subwindow $[10,16]$. 
Figure 1. Equations.

$$
\begin{aligned}
& f(w)=\sum P_{\text {black }}-\sum P_{\text {white }} \\
& i i(x, y)=\sum_{x^{l} \leq x, y^{l} \leq y} i\left(x^{l}, y^{l}\right) \\
& i i(x, y)=f(x, y)+i i(x-1, y)+i i(x, y-1)-i i(x-1, y-1) \\
& \text { res }(x, y)=R(0.299)+G(0.587)+B(0.114) \\
& A \oplus B=\left\{z \mid(B)_{z} \subseteq A\right\} \\
& \text { recall or sensitivity }=\frac{T P}{T P+F N} \\
& \text { specificity }=\frac{T N}{T N+F P} \\
& \text { PR }=\frac{T P}{T P+F P} \\
& \text { accuracy }=\frac{T P+T N}{T P+F P+F N+T N}
\end{aligned}
$$

Figure 2. Haar-like features.

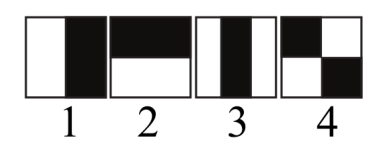

\section{Integral Image}

Integral image is an intermediate representation to quickly calculate the sum of values in a rectangular subset of a grid. The algorithm is used to calculate pixel function in real numbers $f(x, y)$ (ie, to calculate pixel intensity along a rectangular region of the image). For a fast calculation of haar features' rectangles, Viola and Jones [10] proposed the use of integral image (equation b, Figure 1), where $i i(x, y)$ is the integral image and $i(x, y)$ is the original image. For each point $(x, y)$ the value of the sum of all pixels higher and to the left is assigned, such that $x^{1} \leq$ $x, y^{1} \leq y$.

The integral image can be obtained with a single pass over the image, using sum area value added in $(x, y)$ (equation c, Figure $1)$. Once an integral image is computed, the evaluation of any area of the rectangle can be performed in constant time, using only four image references.

\section{Adaptive Boost Algorithm}

Viola and Jones [10] used the idea of weak classifiers, $h(x, f, p, \theta)$. In that definition, there is a simple structure containing a subwindow $(x)$ that consists of a feature $(f)$, threshold $(\theta)$, and parity $(p)$.

The learning algorithm selects a unique feature that best separates the positive and negative examples. This feature was based on a machine learning meta-algorithm proposed by Freund and Schapire [14]. The outputs of the learning algorithm are called weak classifiers. These weak classifiers were combined with a weighted sum that represented the final result of the potential classifier. Its main feature was the distribution of weights in sets of examples and the distribution change over the course of the algorithm iterations [10].

\section{Cascade of Classifiers}

In the learning tasks, a high cost was given for evaluating the entire training set. While the reduction in the number of classifiers tended to improve the speed, the decrease of classifiers reduced the hit hat [15]. Viola and Jones proposed a solution to get a choice of good classifiers in a large set by creating decision trees called cascades of classifiers [10]. In the initial stage, the cascade of classifiers searched the simplest classifiers (generic) and rejected the greatest number of potential negative subwindows. The algorithm began with few features and, as it advanced, the number of cascade stages increased. With more stages the number of classifiers grew and the accuracy improved. In the detection of faces, the best cases are given between 15 to 25 stages [10].

\section{Image Processing}

Image processing techniques seek to improve the appearance or simplify an image, by correcting and/or eliminating noise that arose during image acquisition (equipment) or as a result of image degradations (lighting problems). Domains that are given to highlight on images are space, which refers to the plane of the image working directly on top of the pixels and frequency, which are changes in the images after Fourier transformation [17]. The interpretation of digital data is a computationally 
complex task with a high computational cost [8]. The segmentation or partitioning of digital images into smaller parts is thus an essential task to assist the image interpretation process and images, in general, often contain distinct features that can complicate this process.

Differently-sized images, distorted shapes, lighting problems, and other characteristics contribute to image noise. Image noise, along with certain data contained in images can hamper the identification of features. For example, the image capture of car license plates occurs in uncontrolled environments (ie, lighting variations by virtue of time and weather conditions) [17]. The simplest and most widely used image segmentation technique is binarization which consists of classifying the pixels of a given image according to one or several thresholds. This technique is widely used to separate the background from the objects of interest. In global binarization, cutting a single threshold value is set for the entire image. This is advantageous because it improves processing time; however, image quality may be poor because of potential image noise. As such, global binarization is more suitable for controlled environments. On the other hand, local binarization allows for different cutoff points to different regions of the image. A disadvantage of this technique is the high processing time [14,18].

\section{Experimental Approach}

We developed a mobile device-based automated system to detect malaria. The algorithm specifically identifies Plasmodium falciparum in the trophozoite ring stage. Preprocessing steps were used to improve the quality of the microscopy images (eg, Otsu [19]), and Gaussian filters [20] and mathematical morphology techniques [14] were used for training and classification. With respect to image acquisition, preprocessing, training, and validation were performed on a personal computer (Intel Core i3-3217U $1.8 \mathrm{GHz} 4096 \mathrm{MB}$ RAM) prior to the development of the mobile app (Android 4.2 or higher). The flow of activities in the experimental model is illustrated in Figure 3.

Figure 3. Flowchart of the experimental model.

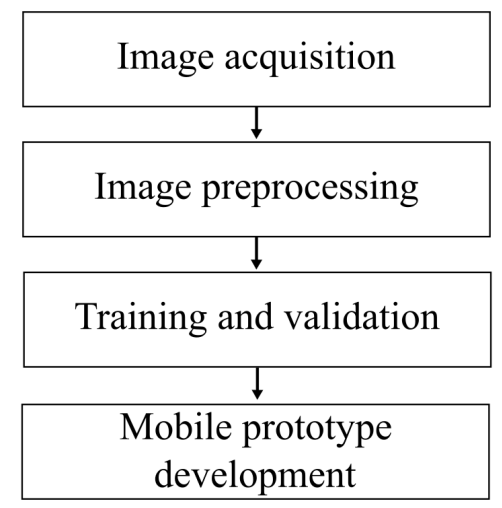

\section{Image Acquisition}

An image acquisition step was necessary in the development of the experimental model. The Microbiology Department (Drassanes Unit) of Vall Hebron Hospital, Barcelona, Spain and the microbiology lab of the Research Center Aggeu Magalhães(FIOCRUZ) Recife, Brazil have a collection of about 500 slides from patients diagnosed with malaria. All the images obtained were classified by experimental parasitologists. This collection was used to perform the image acquisition for the diagnosis algorithm.

Malaria parasites require magnification of at least 1000 times for their identification in blood smears [1]. A light microscopy was used along with the Logitech c270 (webcam), the Samsung Galaxy Tab 2 (mobile phone), and the Sony DSC H1 (semi-professional camera). Polyvinyl chloride (PVC), low-cost support pieces (less than US \$1) were designed to attach the devices to the light microscopes and to the $3 \mathrm{D}$ printer. The image acquisition stage is illustrated in Figure 4. 
Figure 4. Polyvinyl chloride (PVC) was used as support for the tablet (left) and mobile phone (right).

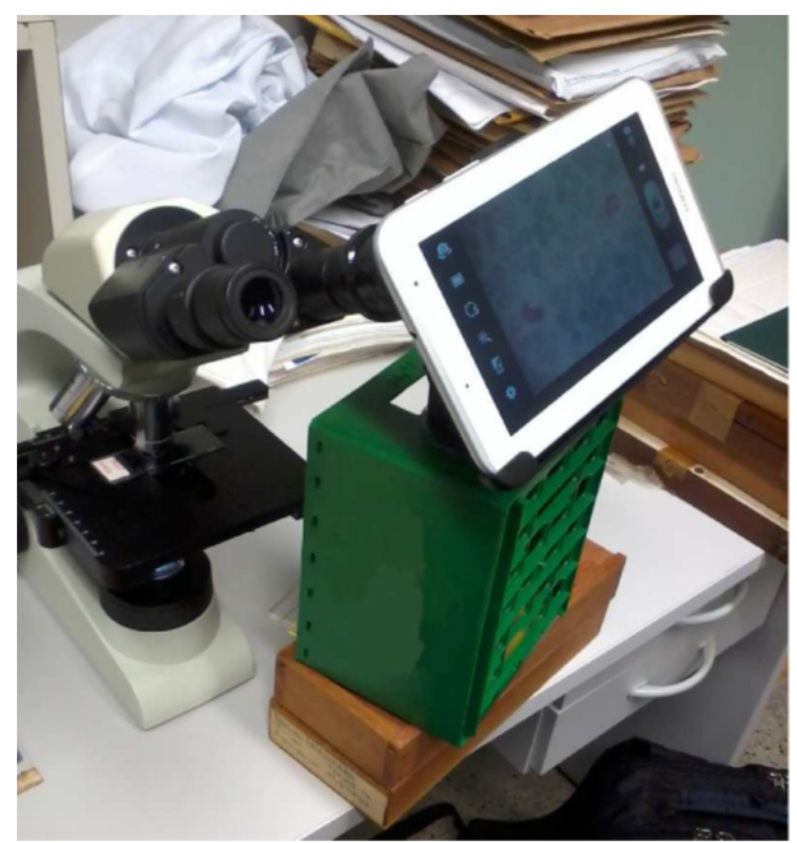

\section{Image Preprocessing}

In the preprocessing step, some improvements in the images were made to limit the scope or search space for the classification algorithm. The preprocessing step is shown in Figure 5. The original image was given in the red, green, blue (RGB) color model. This model was used because it is the most popular and simple to implement. In addition, since RGB is used in devices such as monitors, video cameras, and mobile phones, conversions to other color models was not required [8]. For this step, the original image was acquired through the blood smear method [1].

The objective of this preprocessing step was to reduce the search scope, such as separate blood smears of the background. In this study, we did not use the entire RGB color space model, since performing segmentation operations on images with several layers of colors can be quite expensive computationally [8]. The original image was thus converted to grayscale with only a single color space (equation d, Figure) where res $(x, y)$ is the image in grayscale. After converting the image, the grayscale was replaced by a single color space, with black of lesser intensity and white of greater intensity [9].

A Gaussian filter was then used which gave the convolution, where, for each item on the input array, a Gaussian kernel was applied. The output is the filter operation based in a Gaussian function $[14,19]$. The kernel size was fixed to $7 \times 7$, so that the result was a smoother image. This operation was justified for a better bottom extraction [14], and with the reduction of some details and elements of the images, the edges of the red blood

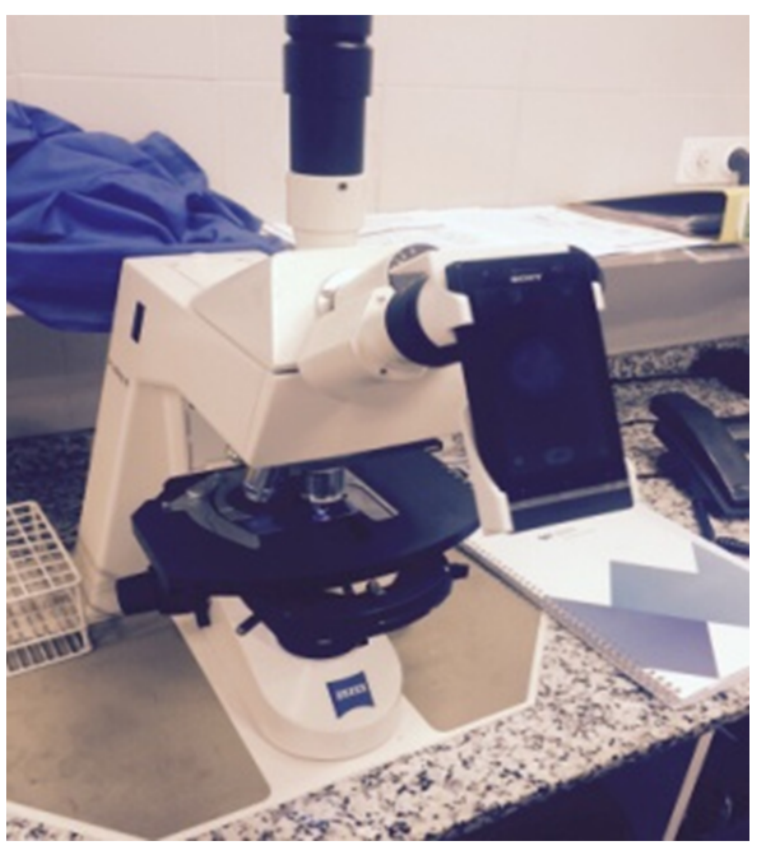

cells became more defined. Thresholding separated the grayscale in a given image such that the image was transformed into two grayscales groups: 0 (100\% black) and 255 (100\% white). For this process, it was necessary to set a cutoff or threshold to separate the two groups. The definition of this cutoff point was very important, because the images had different characteristics (eg, lighting and focus).

The Otsu thresholding technique [19] was chosen because it is widely used in the literature and the cutoff feature has an optimal, automatic threshold. The OTSU technique uses a nonparametric approach (not estimating the parameters of the model) and an unsupervised stop to find the automatic threshold in a given image [21]. The method contains the background and foreground pixel classes, represented by $\mathrm{C} 1$ and $\mathrm{C} 2$, respectively [19]. After the image was smoothed by the Gausssian filter, it was transformed into a binary image containing only foreground (blood cells) and background (rest of the image). With respect to malaria detection, the background is not important because most parasites lie within the cells of the prepared blood smears [5]. Morphological operations may be applied to images to enhance the geometric structures on a set of pixels. Erosion is a morphological technique that seeks to combine two sets by subtracting the vectors for the intersection sets of $\mathrm{A}$ and $\mathrm{B}$ (equation e, Figure 1).

The preprocessing transformation steps were necessary for locating the RBCs and restricting the search space of the algorithm. After the erosion, a subtraction was performed with the image converted to grayscale and the background was removed. The final result is shown in Figure 5. 
Figure 5. Image preprocessing steps.

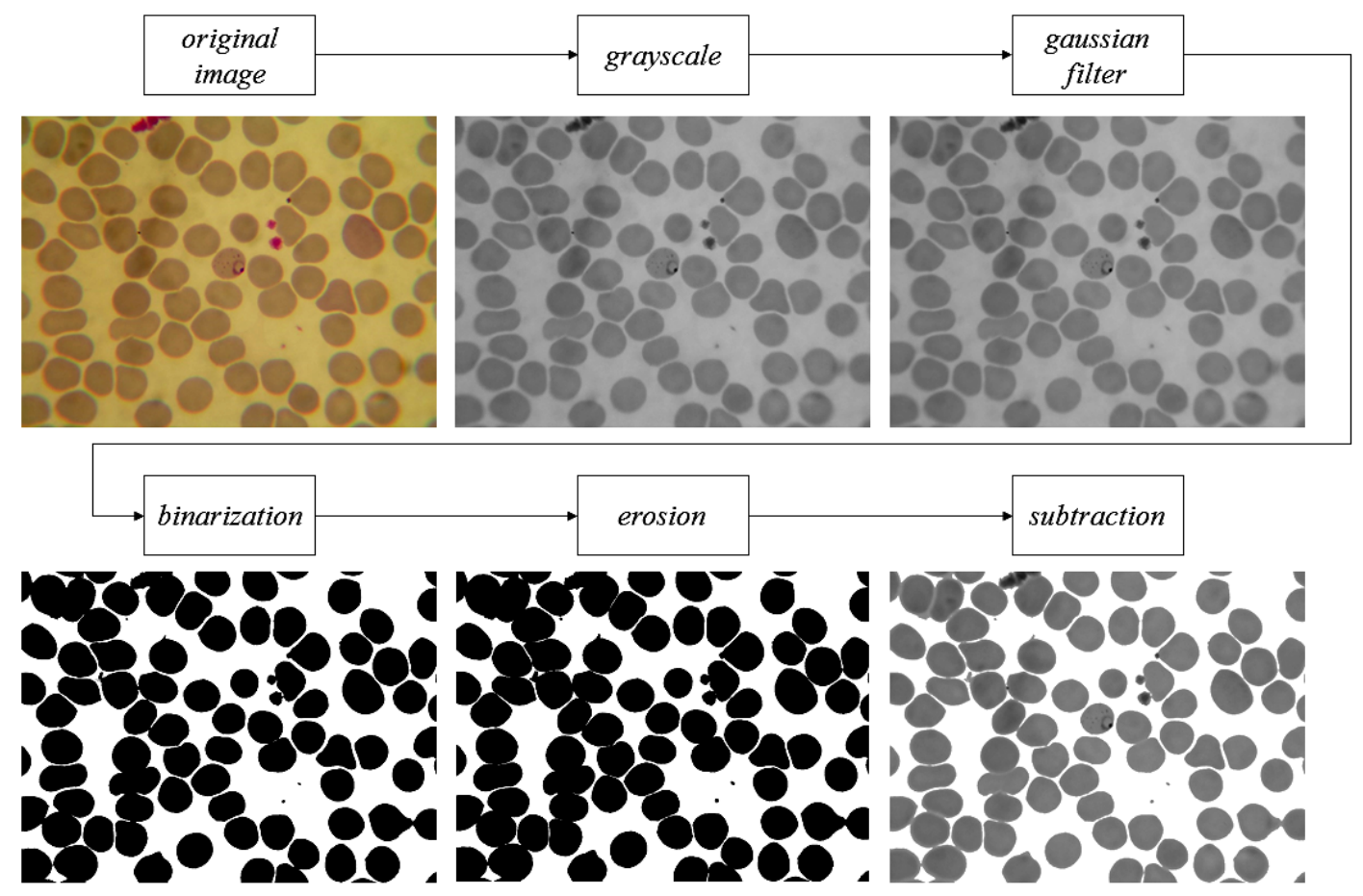

\section{Training and Validation}

The objective of the training and validation steps was to find a good classifier to recognize the parasite Plasmodium falciparum in the trophozoite (ring stage) in a given image. The training and validation of classifiers was performed according to the method by Viola and Jones [10] using an Intel Core i3-3217U (1.8 GHz 4096MB) computer.

For all the training sets, the following parameters were used: (1) the amount of memory was 1024, (2) a minimum hit rate of $0.99500,(3)$ a maximum false alarm rate of $0.400000,(4)$ basic, haar-like features mode [10,22], (5) a subwindow weight of 24, and (6) a subwindow height of 24.

Every image was classified as positive or negative, where positive referred to images infected with the Plasmodium falciparum parasites (trophozoite ring stage), whereas negative referred to white blood cells and empty RBCs (false positive). After classifying the images, the adaptive boosting algorithm was used to select the best features according to the stage of the cascade of classifiers [10]. The result of the training was a degenerative decisions tree with two positive and negative classes.

\section{Mobile App Development}

Currently, the major developers of operating systems for mobile devices are Google Android, Apple IOS, and Microsoft Windows Phone [23-26]. Globally, Android is the most popular mobile operating system with about 1 billion users connected on various devices (tablets, mobile phones, televisions, etc) [24]. Android-based apps are mostly developed in Java [27]; however, apps can be also created in $\mathrm{C} / \mathrm{C}++$ using the Native Development Kit (NDK) [24].

We developed an automated, mobile device-based tool (Android) for the rapid and accurate diagnosis of malaria. The prototype development flow (pseudocode) is shown in Table 1. 
Table 1. Prototype development flow.

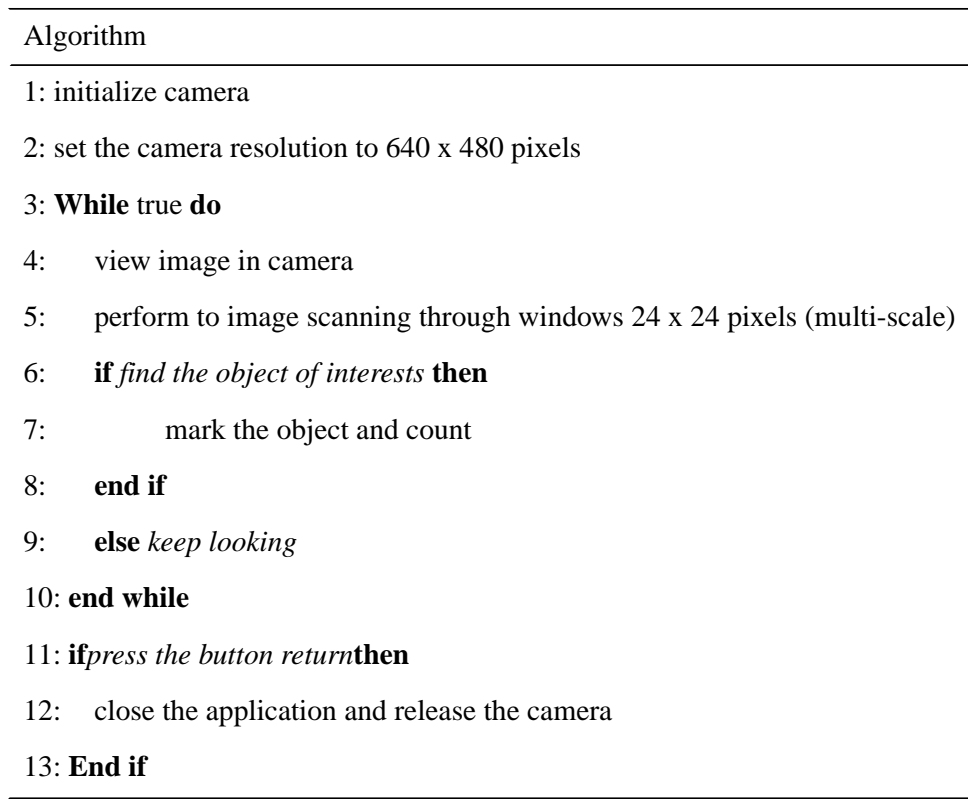

Detection was performed by the mobile camera operating at a resolution of $640 \times 480$ pixels, with an average rate of 5 frames per second (FPS). Factors such as distance, lighting, and training set influenced the detection rate. While the camera is fixed, sight (focus) is adjusted manually on a light microscope. Parasites were detected on a frame-by-frame basis [10].

A second app was also developed that detects malaria in previously-acquired images. While the image analysis procedure is the same, the second app has a button that can upload an image rather than acquiring it with the device camera.

\section{Results}

A set of experiments was designed to evaluate and quantify the accuracy of the developed tool in diagnosing malaria. The evaluation was binary, using positive (infected with Plasmodium-type parasites) and negative (not infected) classes. The acronyms used in the evaluation were positive sample $(\mathrm{P})$, negative sample $(\mathrm{N})$, parasites correctly detected as positive (TP), correctly identified as negative objects (TN), objects that are not parasites of the Plasmodium $s p$ (FP), and positive parasites not detected by the classifier $(\mathrm{FN})$.

In the field of artificial intelligence, metrics to evaluate performance are given. Here, the metrics of recall or sensitivity, specificity, precision rate (PR), and accuracy were used to evaluate the classifiers [28,29]. Recall or sensitivity measured the percentage of positive samples correctly classified as positive samples (equation f, Figure 1). Specificity measured the percentage of correctly identified negative samples out of the total number of negative samples (equation g, Figure 1). PR was the probability that a retrieved sample was relevant (equation h, Figure), whereas accuracy was the proximity degree between the value obtained and the true value (equation i, Figure 1).

Our system was tested using samples from the Microbiology Department (Parasitology Drassanes Unit) of Vall Hebron Hospital, Barcelona, Spain and the (microbiology lab) of Research Center Aggeu Magalhães (FIOCRUZ) Recife, Brazil. For these samples, the images were acquired using the mobile devices (Figure 4). The rest of the images were obtained from Wellcome Images [30,31].

As a preliminary experiment, the classifier was given 555 positive and 777 negative samples. A 10-fold cross-validation procedure was used for training and testing [10] using the adaptive boost algorithm. The results are shown in Tables 2 and 3. 
Table 2. The 10-fold, cross-validation procedure on 10-, 15-, and 20-stage cascades.

\begin{tabular}{|c|c|c|c|c|c|}
\hline Cascade & Iteration $(\mathrm{K})$ & Specificity & Recall or sensitivity & Accuracy & $\mathrm{PR}^{\mathrm{a}}$ \\
\hline \multicolumn{6}{|l|}{ 10-stage } \\
\hline & 1 & 0.7075 & 0.7272 & 0.7096 & 0.2272 \\
\hline & 2 & 0.9331 & 0.6428 & 0.9019 & 0.5373 \\
\hline & 3 & 0.6414 & 0.8070 & 0.6596 & 0.2169 \\
\hline & 4 & 0.8838 & 0.6545 & 0.8596 & 0.4000 \\
\hline & 5 & 0.8731 & 0.7090 & 0.8557 & 0.3979 \\
\hline & 6 & 0.8666 & 0.8727 & 0.8673 & 0.4363 \\
\hline & 7 & 0.3383 & 0.6785 & 0.375 & 0.1101 \\
\hline & 8 & 0.1626 & 0.8983 & 0.2461 & 0.1207 \\
\hline & 9 & 0.6206 & 0.7857 & 0.6384 & 0.2000 \\
\hline & 10 & 0.7960 & 0.8437 & 0.8019 & 0.3673 \\
\hline \multicolumn{6}{|l|}{ 15-stage } \\
\hline & 1 & 0.9548 & 0.5090 & 0.9076 & 0.5714 \\
\hline & 2 & 0.9892 & 0.4000 & 0.9269 & 0.8148 \\
\hline & 3 & 0.9137 & 0.6964 & 0.8903 & 0.4936 \\
\hline & 4 & 0.9634 & 0.7636 & 0.9423 & 0.7118 \\
\hline & 5 & 0.9590 & 0.7142 & 0.9326 & 0.6779 \\
\hline & 6 & 0.9482 & 0.9107 & 0.9442 & 0.6800 \\
\hline & 7 & 0.8134 & 0.7627 & 0.8076 & 0.3435 \\
\hline & 8 & 0.8599 & 0.6607 & 0.8384 & 0.3627 \\
\hline & 9 & 0.8663 & 0.7500 & 0.8538 & 0.4038 \\
\hline & 10 & 0.9234 & 0.7301 & 0.9000 & 0.5679 \\
\hline \multicolumn{6}{|l|}{20 -stage } \\
\hline & 1 & 0.9913 & 0.1636 & 0.9038 & 0.6923 \\
\hline & 2 & 0.9956 & 0.1818 & 0.9096 & 0.8333 \\
\hline & 3 & 0.9698 & 0.4464 & 0.9134 & 0.6410 \\
\hline & 4 & 0.9655 & 0.7500 & 0.9423 & 0.7241 \\
\hline & 5 & 0.9698 & 0.8035 & 0.9519 & 0.7627 \\
\hline & 6 & 0.9590 & 0.8214 & 0.9442 & 0.7076 \\
\hline & 7 & 0.9092 & 0.6491 & 0.8807 & 0.4683 \\
\hline & 8 & 0.9652 & 0.4576 & 0.9076 & 0.6279 \\
\hline & 9 & 0.9202 & 0.7500 & 0.9019 & 0.5316 \\
\hline & 10 & 0.9650 & 0.4677 & 0.9057 & 0.6444 \\
\hline
\end{tabular}

${ }^{\mathrm{a}} \mathrm{PR}$ : precision rate. 
Table 3. Metric results reported as means (SDs).

\begin{tabular}{lll}
\hline Metric & Stage & Mean (SD) \\
\hline Recall or sensitivity $^{\mathrm{a}}$ & 10 & $0.7619851(0.092387416)$ \\
& 15 & $0.6897741(0.142576715)$ \\
Specificity $^{\text {b }}$ & 20 & $0.5491375(0.244642849)$ \\
& 10 & \\
Average accuracy & 15 & $0.6823637(0.2541448)$ \\
& 20 & $0.9191804(0.0558795)$ \\
PR $^{c}$ & 10 & $0.9611227(0.0272376)$ \\
& 15 & $0.6915384(0.2224334)$ \\
& 20 & $0.8938034(0.0497291)$ \\
& 10 & $0.9161538(0.0225766)$ \\
& 20 & $0.3014110(0.1450627)$ \\
& & $0.5627748(0.1602032)$ \\
& & $0.6633560(0.1068479)$ \\
\hline
\end{tabular}

${ }^{\mathrm{a}}$ False positive rate.

${ }^{\mathrm{b}}$ False positive rate.

${ }^{\mathrm{c}} \mathrm{PR}$ : precision rate.

\section{Discussion}

\section{Principal Findings}

We developed a low-cost, automated, mobile device-based tool to diagnose malaria using image segmentation and artificial intelligence techniques. Based on the preliminary results, the classifier algorithm performed better using the 20 -stage cascade with average specificity and accuracy values of $96 \%$ and $91 \%$,

respectively. Recall, however, was better using the 10-stage cascade, even though there were a high number of false positives that decreased the precision of the algorithm. Precision-recall curves were generated for the four best iterations $(\mathrm{K})$ using the cross-validation method (Figure 6). The best area under the precision-recall curve was when recall and precision assumed a value of 1 . After analyzing the curves, it was estimated that the 15-stage cascade in iteration 6 yielded the best result with respect to area under the curve $(70 \%)$.

Figure 6. Precision-recall curve of the four iterations of the cross-validation method.

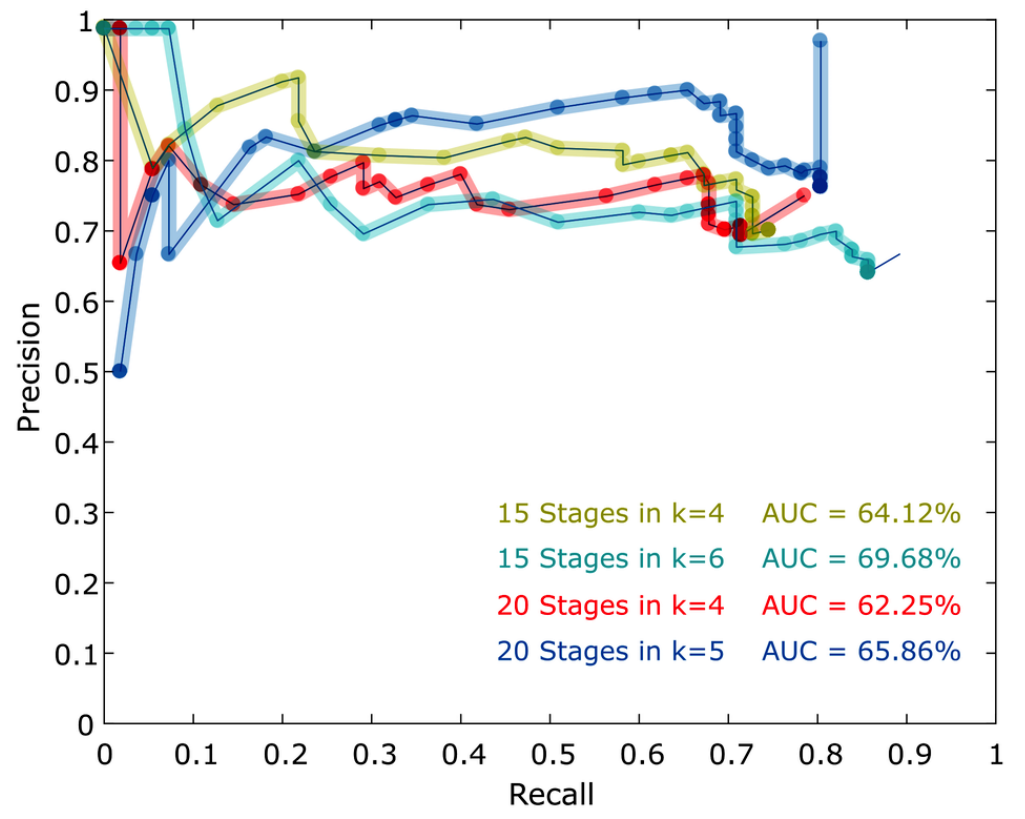




\section{Future Work}

Currently, the highest level of accuracy was 91\%. Based on these findings, the tool is feasible $[12,13]$ and with new sets of positive images, higher rankings with respect to specificity and sensitivity are possible. In the future, variations and adaptations to the Viola and Jones [10] method may be enable detection of other species and stages of malaria development. In addition, new training algorithms such as neural networks [32], support vector machines [33], and even other image processing techniques $[8,20]$ may be explored.

\section{Conclusions}

The development of a low-cost, rapid, and accurate diagnosis tools for mobile phones and tablets that can be used in health centers in remote communities without the need for specific expertise could help break the accessibility barriers of low-resource countries. The tool that we developed can achieve this by virtue of its accessibility and on the spot, real-time diagnostic potential that may facilitate immediate treatment.

\section{Acknowledgments}

This work was partially supported by the Applied Informatics Graduate Program (PPGIA), funded by FACEPE (grant IBPG-1300-1.03/11), and by the National Institute of Science and Technology for Software Engineering (INES), funded by CNPq and FACEPE (grants 465614/2014-0 and APQ-1037-1.03/08).

\section{Conflicts of Interest}

None declared.

\section{Multimedia Appendix 1}

Screenshot of the Malaria System app beta version.

[PDF File (Adobe PDF File), 319KB-Multimedia Appendix 1]

\section{Multimedia Appendix 2}

Video demo of the Malaria System.

[MOV File, 5MB-Multimedia Appendix 2]

\section{References}

1. World Health Organization. World Malaria Report 2015. 2015 Dec. URL: http://www.who.int/malaria/publications/ world-malaria-report-2015/report/en/ [accessed 2016-10-01] [WebCite Cache ID 6kwew4pPq]

2. World Health Organization. Management of Severe Malaria: A Practical Handbook, Third Edition. 2012. URL: http://apps. who.int/iris/bitstream/10665/79317/1/9789241548526_eng.pdf?ua=1[WebCite Cache ID 6png8iL9Z]

3. de Oliveira MR, de Castro Gomes A, Toscano CM. Cost effectiveness of OptiMal® rapid diagnostic test for malaria in remote areas of the Amazon Region, Brazil. Malar J 2010 Oct 11;9:277 [FREE Full text] [doi: 10.1186/1475-2875-9-277] [Medline: 20937094]

4. World Health Organization. Basic Malaria Microscopy: Part 1 Learner's Guide. Geneva: World Health Organization; 1991.

5. World Health Organization. New Perspectives: Malaria Diagnosis. URL: http://www.who.int/tdr/publications/documents/ malaria-diagnosis.pdf [accessed 2016-09-19] [WebCite Cache ID 6ke1kzDx4]

6. Leal Neto OB, Albuquerque CM, Albuquerque JO, Barbosa CS. The schisto track: a system for gathering and monitoring epidemiological surveys by connecting geographical information systems in real time. JMIR Mhealth Uhealth 2014 Mar 10;2(1):e10 [FREE Full text] [doi: 10.2196/mhealth.2859] [Medline: 25099881]

7. Kaewkamnerd S, Uthaipibull C, Intarapanich A, Pannarut M, Chaotheing S, Tongsima S. An automatic device for detection and classification of malaria parasite species in thick blood film. BMC Bioinformatics 2012;13 Suppl 17:S18 [FREE Full text] [doi: 10.1186/1471-2105-13-S17-S18] [Medline: 23281600]

8. Gonzalez RC, Woods RS. Digital Image Processing. Upper Saddle River, NJ: Pearson/Prentice Hall; 2008.

9. Anggraini D, Nugroho A, Pratama C, Rozi I, Iskandar A, Hartono R. Automated status identification of microscopic images obtained from malaria thin blood smears. 2011 Presented at: 2011 International Conference on Electrical Engineering and Informatics (ICEEI); 2011 July 17-19; Bandung, Indonesia p. 6. [doi: 10.1109/ICEEI.2011.6021762]

10. Viola P, Jones MJ. Robust real-time face detection. Int J Comput Vision 2004 May;57(2):137-154. [doi: 10.1023/B:VISI.0000013087.49260.fb]

11. Firmo AC, Oliveira AD, Viegas J, Albuquerque J. Schistosystem - inteligencia artificial para diagnostico automatico por imagens. Porto Alegre, Brazil: IADIS; 2013 Presented at: Atas da Conferencia IADIS Ibero-Americana Computacao Aplicada; 2013 Dec 10-11; Lisbon, Portugal p. 87. 
12. de Oliveira AD, Cabral G, López D, Firmo C, Serrat ZF, Albuquerque JO. A proposal for automatic diagnosis of malaria: extended abstract. 2013 Presented at: Proceedings of the 22nd International Conference on World Wide Web (WWW '13 Companion); 2013 May 13-17; Rio de Janeiro, Brazil p. 681-682. [doi: 10.1145/2487788.2488022]

13. de Oliveira AD, Arruda ME, Prat JG, Codina DL, Serrat FZ, Albuquerque JO. Malaria system: a new tool for automatic diagnosis of malaria in mobile devices. : JMIR; 2014 Presented at: 7th World Congress Medicine 2.0; 2014 Oct 9-10;

Malaga, Spain p. 1 URL: http://www.medicine20congress.com/ocs/index.php/med/med2014b/paper/view/2487

14. Freund Y, Schapire RE. Journal of JSAI. 1999. A short introduction to boosting URL: http://www.yorku.ca/gisweb/eats4400/ boost.pdf [accessed 2016-09-20] [WebCite Cache ID 6kfHYe9Si]

15. Gonzalez RC, Woods RE. Processamento de Imagens Digitais. São Paulo, Brazil: Edgard Blucher; 2000:85-212.

16. Papageorgiou C, Oren M, Poggio T. A general framework for object detection. 1998 Presented at: IEEE International Conference on Computer Vision; 1998 Jan 7; Bombay, India. [doi: 10.1109/ICCV.1998.710772]

17. Pedrini H, Schwartz W. Análise de Imagens Digitais: Princípios, Algoritmos e Aplicações. São Paulo, Brazil: Thomson Learning; 2008:506.

18. Bradski G, Kaehler A. Learning OpenCV: Computer Vision With the OpenCV Library. Sebastopol, CA: O'Reilly; 2008.

19. Otsu N. A threshold selection method from gray-level histograms. IEEE Trans Syst Man Cybern 1979 Jan;9(1):62-66. [doi: 10.1109/TSMC.1979.4310076]

20. Szeliski R. Computer Vision: Algorithms and Applications (Texts in Computer Science) 2011th Edition. Springer London: Springer; 2011.

21. Laganière R. OpenCV 2 Computer Vision Application Programming Cookbook. Ottawa: Packt Publishing Limited; 2011.

22. Lienhart R, Maydt J. An extended set of haar-like features for rapid object detection. Piscataway, New Jersey: IEEE; 2002 Presented at: ICIP: 2002 International Conference on Image Processing; 2002 Sept 22-25; Rochester.

23. Android Developer. URL: https://developer.android.com/index.html [accessed 2016-09-20] [WebCite Cache ID 6kfIHUOUN]

24. Mednieks Z, Dornin L, Meike G, Nakamura M. Programming Android: Java Programming for the New Generation of Mobile Devices. California: O'Reilly Media; 2012.

25. IOS Developer. URL: https://developer.apple.com/ [accessed 2016-09-20] [WebCite Cache ID 6kfItR0Ek]

26. Microsoft Windows Phone. URL: https://www.microsoft.com/windows/phones [accessed 2016-09-20] [WebCite Cache ID 6kfIzUwOV]

27. Schildt H, Skrien D. Programação com Java: Uma Introdução Abrangente. São Paulo, Brazil: Bookman Editora; 2013.

28. Olson DL, Delen D. Advanced Data Mining Techniques. Berlin: Springer; 2008.

29. Araujo GM. Algoritmo Para Reconhecimento de Características Faciais Baseado em Filtros de Correlação. Master's thesis. PPEE - UFRJ. 2010. URL: http://www02.smt.ufrj.br/ eduardo/teses/gabriel-matos-mestrado.pdf [accessed 2016-09-20] [WebCite Cache ID 6kfJOJJf7]

30. Center for Disease Control Prevention. Public Health Image Library (PHIL). URL: http://phil.cdc.gov/phil/default.asp [accessed 2016-09-20] [WebCite Cache ID 6kfJVUdyl]

31. Wellcome Images. URL: https://wellcomeimages.org/ [accessed 2016-09-20] [WebCite Cache ID 6kfJaKLQJ]

32. Haykin SS. Redes Neurais: Princípios e Práticas. São Paulo, Brazil: Bookman Companhia Ed; 2001.

33. Duda RO, Hart PW, Stork DG. Pattern Classification. New York: John Wiley \& Sons; 2001.
Abbreviations
FPR: false positives rate
PR: precision rate
RBCs: red blood cells
RCT: randomized controlled trial
RDT: rapid diagnostic tests
RGB: red, green, blue
ROC: receiver operating characteristic
TPR: true positives rate 
Edited by G Eysenbach; submitted 06.10.16; peer-reviewed by F Ofli, S Chen, K Gary; comments to author 25.11.16; revised version received 07.01.17; accepted 04.03.17; published 25.04.17

Please cite as:

Oliveira AD, Prats C, Espasa M, Zarzuela Serrat F, Montañola Sales C, Silgado A, Codina DL, Arruda ME, i Prat JG, Albuquerque $J$

The Malaria System MicroApp: A New, Mobile Device-Based Tool for Malaria Diagnosis

JMIR Res Protoc 2017;6(4):e70

URL: http://www.researchprotocols.org/2017/4/e70/

doi: $10.2196 /$ resprot.6758

PMID: 28442456

(C)Allisson Dantas Oliveira, Clara Prats, Mateu Espasa, Francesc Zarzuela Serrat, Cristina Montañola Sales, Aroa Silgado, Daniel Lopez Codina, Mercia Eliane Arruda, Jordi Gomez i Prat, Jones Albuquerque. Originally published in JMIR Research Protocols (http://www.researchprotocols.org), 25.04.2017. This is an open-access article distributed under the terms of the Creative Commons Attribution License (http://creativecommons.org/licenses/by/2.0/), which permits unrestricted use, distribution, and reproduction in any medium, provided the original work, first published in JMIR Research Protocols, is properly cited. The complete bibliographic information, a link to the original publication on http://www.researchprotocols.org, as well as this copyright and license information must be included. 